\title{
Deshidratado de Physalis peruviana L. en dos Estados de Madurez y su Efecto sobre el Contenido de Polifenoles Totales, Capacidad Antioxidante, Carotenos, Color y Ácido Ascórbico
}

\author{
Juan C. De la Vega*, Magali A. Cañarejo, Omar N. Cabascango y Marco V. Lara \\ Universidad Técnica del Norte, Facultad de Ciencias Agropecuarias y Ambientales, Grupo de investigación en Desarrollo \\ e Innovación Bioalimentaria y Nutracéutica, GIDIBAN, Imbabura, Ecuador \\ (e-mail: jcdelavega@utn.edu.ec; macanarejo@utn.edu.ec; oncabascangom@utn.edu.ec; mvlara@utn.edu.ec)
}

* Autor a quien debe ser dirigida la correspondencia

Recibido Oct. 24, 2018; Aceptado Dic. 10, 2018; Versión final Ene. 23, 2019, Publicado Oct. 2019

\begin{abstract}
Resumen
El presente trabajo investigó el contenido de polifenoles totales, carotenos, ácido ascórbico, capacidad antioxidante y el color de Physalis peruviana L. al ser deshidratada a $60^{\circ} \mathrm{C}$ mediante secador de bandejas a una velocidad de $3 \mathrm{~m} / \mathrm{s}$ y mediante estufa a la misma temperatura. El contenido de polifenoles totales se evaluó con el método de Folin-Ciocalteu y la capacidad antioxidante por el método 2,2-Difenil-1-Picrilhidrazilo (DPPH), el color mediante CIELab, carotenos por espectrofotometría y ácido ascórbico por titulación. Se encontró que la fruta presentó una capacidad antioxidante de 237.3 y $260.7 \mu \mathrm{molTE} / 100 \mathrm{~g}$, un contenido de polifenoles totales de 129.90 y $124.86 \mathrm{mg} \mathrm{GAE} / 100 \mathrm{~g}$, un contenido de carotenos de 136.96 y $149.26 \mu \mathrm{g} / \mathrm{g}$, mientras que el contenido de ácido ascórbico fue de 21.03 y $19.10 \mathrm{mg} / 100 \mathrm{~g}$ para fruta de madurez 3 y 5 respectivamente. La capacidad antioxidante presentó una correlación inversa con el contenido de polifenoles y ácido ascórbico, pero directa con el de carotenos. La mayor conservación de las propiedades estudiadas, especialmente en los carotenos, se observó con el secador de bandejas, por lo que se recomienda este método de procesamiento de deshidratación.
\end{abstract}

Palabras clave: actividad antioxidante; polifenoles totales; carotenos; fruta deshidratada

\section{Dehydrated Physalis peruviana L. in Two Ripening States and its Effect on Total Phenolic Compounds, Antioxidant Capacity, Carotenes, Color and Ascorbic acid}

\begin{abstract}
This document aims to research about total phenolic compounds, carotenes, ascorbic acid, antioxidant capacity and color of Physalis peruviana L. after been dehydrated at $60^{\circ} \mathrm{C}$ by tray dryer with velocity of $3 \mathrm{~m} / \mathrm{s}$ and heater. For total phenolic compounds evaluation, the Folin-Ciocalteu method was used and the antioxidant capacity was determined by the method 2,2-diphenyl-1-picrylhydrazyl (DPPH), the CIELab scale was used for color and spectrophotometry for carotenes, while ascorbic acid was measure by titration. The results indicated that the fruit has an antioxidant capacity of 237.3 and $260.7 \mu \mathrm{molTE} / 100 \mathrm{~g}$, a concentration of total polyphenols of 129.90 and $124.86 \mathrm{mg} \mathrm{GAE} / 100 \mathrm{~g}, 136.96$ and $149.26 \mu \mathrm{g} / \mathrm{g}$ carotene content and 21.03 and $19.10 \mathrm{mg} / 100$ $\mathrm{g}$ ascorbic acid content for ripeness of 3 and 5 respectively. The antioxidant capacity presented an inverse correlation with the content of polyphenols and ascorbic acid, but directly with that of carotenoid. The higher conservation of the properties studied, especially in the carotenes, was observed with the tray dryer, so this method of processing is recommended for dehydration.
\end{abstract}

Keywords: antioxidant capacity; phenolic compounds; carotenes; dehydrated fruit 


\section{INTRODUCCIÓN}

La deshidratación es una de las técnicas más comunes en la industria para conservar alimentos o producir snacks, aumentando su vida útil tras la disminución de la humedad, utilizando equipos como estufas y secadores de bandeja (Peinado et al., 2013, Giraldo et al., 2009). Sin embargo, este proceso afecta considerablemente los componentes bioactivos (polifenoles, vitamina $\mathrm{C}$, entre otros) y las propiedades funcionales (capacidad antioxidante) que puedan existir en los alimentos, debido a las temperaturas de deshidratación y al tiempo de tratamiento. Las frutas particularmente poseen varios componentes bioactivos beneficiosos para la salud humana, entre ellos se encuentran la vitamina A, B1, B2, B3, B6, B12, C, E, polifenoles y otros (Teixeira et al. 2016), que les otorgan propiedades nutricionales, medicinales y funcionales cada vez más relevantes para el consumidor, lo que ha motivado múltiples investigaciones con la finalidad de incrementar el tiempo de consumo, conservando su valor funcional (Scalzo et al., 2005).

La importancia de evaluar la capacidad antioxidante en una fruta radica en que se asocia a los compuestos que poseen la habilidad de suprimir los efectos perjudiciales causados por las especies reactivas de oxígeno (radicales libres) (Solorzano et al., 2015, Franco et al., 2017). Los radicales libres son sustancias que poseen uno o más electrones desapareados, convirtiéndolos en compuestos altamente reactivos que provocan el estrés oxidativo (desbalance entre los compuestos oxidantes y los antioxidantes). Este estrés oxidativo puede generar daños en el ADN de las células, en las proteínas, carbohidratos, aminoácidos, etc. Los antioxidantes minimizan este efecto pues reaccionan con los radicales libres antes de que alcancen los compuestos biológicos. Esta habilidad de capturar un radical se conoce como capacidad antioxidante, y por lo general lo realizan compuestos como la vitamina $C$ (reduce la incidencia de enfermedades degenerativas), la vitamina E (agente protector contra la peroxidación lipídica de las membranas), carotenoides (influyen positivamente ante algunos tipos de cáncer) y polifenoles (previenen enfermedades cardiovasculares entre otras) (Hoyos et al., 2017, Cerón et al., 2010, Copello et al., 2018). El método que utiliza la reducción del radical DPPH (1,1difenil-2-picril-hidrazilo) es uno de los más utilizados para evaluar capacidad antioxidante, y consiste en monitorear el decaimiento de la absorbancia a una determinada longitud de onda (Del'Arco y de Sylos, 2018).

Los polifenoles son grupos representativos de metabolitos secundarios y se consideran compuestos biológicamente activos que no son nutrientes y en ocasiones pueden contribuir con propiedades organolépticas (sabor o color) de las frutas (Franco et al., 2017). Los compuestos fenólicos tipo flavonoides pueden generar distintos beneficios aparte de apoyar la capacidad antioxidante que pueda presentar una fruta, también pueden desarrollar actividad antimicrobiana y antiviral, inhibir enzimas, quelar hierro, regular la expresión génica entre otras (Cortés et al., 2015). Los carotenos también pueden relacionarse con el color de las frutas (Puente et al., 2011), la cuantificación de estos compuestos y la evaluación del color de puede ampliar el panorama que determine la capacidad antioxidante.

Este estudio busca comparar los efectos que la deshidratación mediante estufa y secador de bandejas generan en componentes bioactivos (polifenoles, carotenos y ácido ascórbico), la capacidad antioxidante y el color de la fruta Physalis peruviana L., en dos diferentes estados de madurez. Para cumplir este objetivo es necesario conocer el contenido funcional de la fruta Physalis peruviana $\mathrm{L}$. y de la literatura se sabe que su fruto contiene polifenoles, carotenos (3000 IU por $100 \mathrm{~g}$ ), vitamina C (entre 20 y $43 \mathrm{mg}$ por $100 \mathrm{~g}$ de fruto), vitamina $\mathrm{E}$, algunos componentes del complejo B (tiamina, riboflavina, niacina), así como fibra, proteína y fósforo (Teixeira et al., 2016). También se le ha dado propiedades benéficas anticancerígenas y contribución a disminuir los niveles de glucosa y colesterol en la sangre (Reyes-Beltrán et al., 2015). En cuanto a los compuestos fenólicos la fruta presenta en base al método de Folin-Ciocalteu valores desde $145.22 \mathrm{mg}$ Equivalente de ácido gálico (GAE)/ $100 \mathrm{~g}$ de fruto hasta $240.16 \mathrm{mg} \mathrm{GAE} / 100 \mathrm{~g}$ de fruto (Teixeira et al., 2016; Yıldız et al. 2015; Mier y Cáez, 2011). Mientras que la capacidad antioxidante oscila entre $192.51 \mu \mathrm{mol}$ TE/100 $\mathrm{g}$ hasta $210.82 \mu \mathrm{mol}$ TE/100 $\mathrm{g}$ en base al método DPPH (Puente et al., 2011).

\section{MATERIALES Y MÉTODOS}

A la materia prima se evaluó el contenido de polifenoles totales, la capacidad antioxidante, carotenos, ácido ascórbico, color, acidez titulable y sólidos solubles (los dos últimos para definir el estado de madurez). Se utilizaron frutos recolectados en la ciudad de Cayambe (Pichincha, Ecuador), cultivados por la organización Resak. La madurez se evaluó inicialmente en campo, seleccionando los frutos con las mejores características físicas y se clasificaron de forma visual mediante la tabla de color (en los estados de madurez 3 para frutos anaranjados con visos verdes hacia la zona del cáliz y 5 para frutos anaranjados), luego en campo se determinó el contenido de sólidos solubles según la Norma Técnica Colombiana (NTC 4580), y posteriormente se evaluó su acidez titulable en laboratorio.

Para la deshidratación se utilizaron $500 \mathrm{~g}$ de muestra que fue lavada y desinfectada con hipoclorito de sodio a 70 ppm por 5 minutos (una muestra aparte se utilizó para realizar los análisis respectivos de la materia 
prima). Posteriormente, se colocó la fruta entera sin cáliz en una bandeja distribuida uniformemente y se introdujo en la estufa, donde se procesó durante 52 horas continuas (alcanzando humedad de equilibrio) a una temperatura de $60{ }^{\circ} \mathrm{C}$. Una segunda muestra de $500 \mathrm{~g}$ se introdujo en el secador de bandejas bajo las mismas condiciones con una velocidad del aire de $3 \mathrm{~m} / \mathrm{s}$ durante 14 horas continuas (igualmente hasta alcanzar la humedad de equilibrio). Este procedimiento se realizó tanto para la fruta en madurez 3 como para la fruta en madurez 5.

\section{Reactivos y equipos}

Los reactivos utilizados para la medición de las variables del presente estudio fueron el 1,1-difenil-2-picrilhidrazilo (DPPH), el ácido-6-hidroxi-2,5,7,8-tetrametilcroman-2-carboxílico (Trolox) y el Folin-Ciocalteu 2N obtenidos de Sigma-Aldrich® (St. Louis, MO, USA). Se utilizó adicionalmente metanol al 99.5\%, ácido3,4,5trihidroxibenzóico (ácido gálico) y carbonato de sodio al 19\% obtenidos de Loba Chemie. Se utilizó un espectrofotómetro de UV-vis Jenway (modelo 6705 UV/Vis), liofilizador modelo Advantage plus ES-53, molino eléctrico Hamilton Beach y un colorímetro Shimadzu 2201.

\section{Preparación de muestra de fruto fresco y deshidratado}

El fruto fresco se congeló a $-15^{\circ} \mathrm{C}$ para facilitar el triturado con un mortero. La muestra triturada se colocó en bandejas de aluminio en forma de capa fina con espesor no mayor a $5 \mathrm{~mm}$, y se liofilizó por aproximadamente 24 horas. Posteriormente la muestra liofilizada se molió en mortero, se empacó y se colocó en desecador. Luego de aproximadamente una hora se prepararon los extractos. El fruto deshidratado se molió en un molino eléctrico Hamilton Beach, se empacó y se colocó en desecador, después de 30 minutos se prepararon los extractos.

\section{Preparación de extractos de fruto fresco y deshidratado}

Los extractos fueron preparados a partir de $0.5 \mathrm{~g}$ de muestra liofilizada (para fruto fresco) o deshidratada y molida (para fruto procesado) disueltos en $10 \mathrm{ml}$ de metanol. La extracción se realizó con agitación durante 15 minutos a $750 \mathrm{rpm}$ y luego se almacenó durante 24 horas a $2{ }^{\circ} \mathrm{C}$ en ausencia de luz. En seguida los extractos fueron centrifugados por 20 minutos a $5000 \mathrm{rpm}$ y filtrados en papel whatman número 4 . Los extractos finales fueron almacenados a $2^{\circ} \mathrm{C}$ para luego de media hora realizar la cuantificación.

\section{Determinación de polifenoles totales}

El contenido de polifenoles totales de los extractos fue determinado acorde al método descrito por Yıldız et al. (2015) con algunas modificaciones. Previo a realizar la cuantificación se preparó una curva de calibración con ácido gálico en concentraciones en el rango de $10-50 \mathrm{ppm}$. Para preparar la muestra se tomó $0.25 \mathrm{ml}$ de extracto, se diluyó con $2.3 \mathrm{ml}$ de agua destilada, luego se agregó $0.15 \mathrm{ml}$ de Folin Ciocalteu al $1 \mathrm{~N}$ y se mezcló en un vortex por 3 segundos. Se dejó reposar a temperatura ambiente por 5 minutos y se adicionó $0.6 \mathrm{ml}$ de carbonato de sodio al 19\%, se agitó la mezcla por 15 segundos y se dejó incubar a temperatura ambiente por 90 minutos en ausencia de luz. La absorbancia de la mezcla fue determinada a $760 \mathrm{~nm}$, utilizándose metanol para calibración del equipo. Los resultados fueron expresados como miligramos equivalentes de ácido gálico (GAE) en $100 \mathrm{~g}$ de fruto seco.

\section{Determinación de capacidad antioxidante}

Para determinar la capacidad antioxidante se utilizó el método DPPH descrito por Corrales et al. (2015) con mínimas modificaciones. Previo a realizar la cuantificación se preparó una curva de calibración de Trolox en concentraciones de 0-60 $\mu \mathrm{M}$. Posteriormente se tomó $20 \mu \mathrm{l}$ de extracto al que se le adicionó $280 \mu \mathrm{l}$ del reactivo DPPH, la mezcla se dejó reposar por 30 minutos en ausencia de luz a temperatura ambiente. La absorbancia se leyó a una longitud de onda de $517 \mathrm{~nm}$ y los resultados fueron expresados como $\mu \mathrm{mol}$ equivalentes de Trolox (TE) / $100 \mathrm{~g}$ de fruto.

\section{Determinación de carotenos}

Para determinar los carotenos se utilizó el método expuesto por Amaya y Kimura (2004) con algunas modificaciones. Se pesó la muestra y se agregó solución de extracción de 50/25/25 (hexano/acetona/etanol, $\mathrm{v} / \mathrm{v} / \mathrm{v}$ ). La solución se centrifugó y se filtró, se repitió el proceso hasta extraer todo el contenido para luego lavar la solución de extracción con agua. Luego se separó en un embudo de decantación y la fase hexánica se aforó y se cuantificó usando la ecuación 1, donde $A$ es la absorbancia leída a $450 \mathrm{~nm}$ del extracto, $V$ es el volumen aforado de la fase hexánica y A1 es el coeficiente de absorción del $\beta$-caroteno en hexano (2592). Los datos se expresan en $\mu \mathrm{g} / \mathrm{g}$. 
contenido total de carotenos $=\frac{A^{*} V(m l) * 10^{4}}{A 1^{*} \text { masa muestra }(g)}$

\section{Determinación de ácido ascórbico}

Para determinar el contenido de ácido ascórbico se utilizó el método de titulación con 2,6-dicloroindofenol de la AOAC 967.21 con ligeras modificaciones. Las muestras se trituraron con un mortero, se pesaron $5 \mathrm{~g}$ y se diluyeron en $10 \mathrm{ml}$ de solución de extracción de ácido meta fosfórico-ácido acético (120 g de ácido meta fosfórico en $750 \mathrm{ml}$ de ácido acético diluido a $2000 \mathrm{ml}$ con agua destilada). La solución se sometió a agitación con ultrasonido durante 20 min a temperatura ambiente, para luego aforar el sobrenadante a $50 \mathrm{ml}$ con solución de extracción. Se tomó una alícuota de $5 \mathrm{ml}$ y se tituló con la solución de indofenol. La solución de titulación se preparó con 20 mg de 2,6-dicloroindofenol y 16.8 mg de Bicarbonato de Sodio en $100 \mathrm{ml}$ de agua destilada. Posteriormente, se tituló el blanco y solución estándar de $10 \mathrm{mg}$ de ácido ascórbico en $10 \mathrm{ml}$ de solución de extracción. Los datos se expresaron en mg/100 g peso fresco.

\section{RESULTADOS Y DISCUSIÓN}

Los resultados se presentan por cada análisis (contenido de polifenoles totales, capacidad antioxidante, ácido ascórbico, color y carotenos) evaluado en el fruto fresco en estado de madurez de 3 y 5 y luego de procesarlos en secador de bandeja y estufa. En todos los casos los resultados se expresan en valor promedio \pm desviación estándar.

\section{Contenido de polifenoles totales}

Para determinar el contenido de polifenoles totales se realizó una curva de calibración utilizando estándar de ácido gálico. La curva se representó mediante la ecuación 2 donde Abs es la absorbancia leída del espectrofotómetro y [ ] es la concentración expresada en ppm. Dicha ecuación presentó un $\mathrm{R}^{2}$ de 0.9917 y un error típico de 1.66 .

\section{[ ] $=61.369^{*}$ Abs-6.1834}

La tabla 1 muestra los resultados del contenido de polifenoles totales de la fruta de estudio, a los cuales se realizó análisis estadístico con Infostat. Las letras minúsculas refieren al análisis de la fruta fresca, mientras que las letras mayúsculas refieren al análisis de las combinaciones de madurez y procesamiento.

Tabla 1: Contenido de polifenoles totales (mg GAE/100 g) de Physalis peruviana L. antes y después de la deshidratación. Los valores que no comparten la misma letra son diferentes, $\mathrm{p}<0.05$.

\begin{tabular}{|l|c|c|}
\hline \multicolumn{1}{|c|}{ Condición } & Estado de madurez 3 & Estado de madurez 5 \\
\hline Fruta fresca & $129.90 \pm 0.47 \mathrm{~b}$ & $124.86 \pm 0.94 \mathrm{a}$ \\
\hline Fruta deshidratada por estufa & $36.58 \pm 0.78 \mathrm{C}$ & $31.23 \pm 1.11 \mathrm{D}$ \\
\hline Fruta deshidratada por secador de bandeja & $62.51 \pm 1.46 \mathrm{~A}$ & $51.91 \pm 2.45 \mathrm{~B}$ \\
\hline
\end{tabular}

De la tabla 1 se puede observar que existe en promedio una degradación alta $(>70 \%)$ de los polifenoles ante el procesamiento de deshidratación por estufa, en comparación con el obtenido al deshidratar por secador de bandeja (>50\%). La pérdida de los compuestos fenólicos se debe a las reacciones de oxidación, tanto enzimáticas como no enzimáticas que se generan durante todo el intervalo de tiempo en el que la fruta se ve sometida al proceso de deshidratación (Nunes et al., 2016).

Entre las reacciones enzimáticas se encuentran las catalizadas por la polifenol oxidasa, que en presencia de oxígeno molecular realiza dos reacciones, la hidroxilación de monofenoles a o-difenoles y la posterior oxidación de o-difenoles a o-quinonas. Estas últimas son moléculas altamente electrofílicas que pueden reaccionar con aminoácidos o proteínas para formar polímeros marrones que propician el pardeamiento de las frutas. La evaluación del color en esta investigación permite apoyar este comportamiento, dada la evidencia en el cambio del color de la fruta durante su procesamiento, al observar y cuantificar un color más intenso (Carriel et al., 2014, Bravo y Osorio, 2016). Además, la degradación se debe a que entre los principales compuestos fenólicos presentes en la fruta Physalis peruviana L. se encuentran la rutina, miricetina, quercetina, epicatequina, catequina y Kaempferol (Sathyadevi y Subramanian, 2015; Giraldo et al., 2017), ácidos fenólicos como cafeico, clorogénico, ferúlico, $p$-cumarico y gálico. De estos se conoce que la quercetina es susceptible a tratamientos térmicos, así como la miricetina y el kaempferol siendo estos dos últimos además susceptibles a la oxidación (Wang et al., 2016). 
La degradación de los polifenoles también se ha observado en otros estudios como en el secado de guayaba para producir pasta de guayaba, donde también se promovió la pérdida de componentes fenólicos durante el secado por estufa, conservando un 60\% (Nunes et al., 2016). El mismo comportamiento se obtuvo en el secado por estufa de calabazas, piel de uvas, tomates, jengibre y frutas cítricas (Aydin y Gocmen, 2015; Que et al., 2008; de Torres et al., 2010; Gümüşay et al., 2015; Sun et al., 2015). En todos los casos, el tiempo de procesamiento al que fue sometido la fruta, hortaliza o tubérculo influyó en la degradación de los polifenoles totales. En la estufa se requirió un mayor tiempo de procesamiento y se evidencia un menor contenido de polifenoles totales, más aún, existe diferencia significativa en estos resultados, y de la tabla 1 se observa que el método en donde se aplica secado por bandejas a la fruta en madurez 3, presenta el mayor contenido de polifenoles, siendo este completamente diferente a los otros tratamientos.

Por otro lado, el contenido de polifenoles totales presente en ambos estados de madurez de la fruta fresca es ligeramente inferior al reportado por Mier y Cáez (2011) que fue de $131.19 \mathrm{mg} \mathrm{GAE} / 100 \mathrm{~g}$ y al de Yıldız et al. (2015) que encontraron un contenido de fenoles totales de 136.64 a $154.55 \mathrm{mg} \mathrm{GAE} / 100 \mathrm{~g}$. Sin embargo, los resultados obtenidos, sí se encuentran en el rango reportado por Teixeira et al. (2016) el cual está entre 106 y $149.3 \mathrm{mg} \mathrm{GAE} / 100 \mathrm{~g}$. Se observa también que existe una diferencia significativa en el contenido de polifenoles totales entre la fruta fresca en madurez 3 y la de madurez 5 , con un comportamiento donde a mayor madurez, menor contenido de polifenoles. El decremento en el contenido de polifenoles en la fruta durante su maduración puede deberse a que los fenoles insolubles se transforman por polimerización, oxidación o la combinación de los dos a ácidos fenólicos ligados en estados que no pueden apreciarse por espectrofotometría e inclusive por HPLC-MS (Gruz et al., 2011). Adicionalmente, la enzima polifenol oxidasa también presenta mayor actividad durante la maduración, lo que incentiva también la transformación de los polifenoles, este comportamiento ha sido reportado en investigaciones anteriores (Bolívar-Fernández et al., 2011).

\section{Capacidad Antioxidante}

Para determinar la capacidad antioxidante se realizó una curva de calibración utilizando estándar de trolox. La curva se representó mediante la ecuación 3 donde Abs es la absorbancia leída del espectrofotómetro y [ ] es la concentración expresada en ppm. Dicha ecuación presentó un $\mathrm{R}^{2}$ de 0.9897 y un error típico de 2.40.

$$
[\quad]=531.73-1965.4^{*} \mathrm{Abs}
$$

La tabla 2 muestra los resultados de la capacidad antioxidante encontrados para el fruto de estudio en estado fresco en sus dos estados de madurez, así como la capacidad antioxidante luego de ser deshidratado por estufa y por secador de bandeja. En esta y en las siguientes secciones al igual que en el caso de los polifenoles se realizó el análisis estadístico comparando los estados de madurez de la fruta fresca y por otro lado las combinaciones de procesamiento y madurez. Las letras minúsculas refieren al análisis de la fruta fresca y las mayúsculas al análisis de los tratamientos.

Tabla 2: Capacidad antioxidante ( $\mu \mathrm{mol}$ TE/100 g) de Physalis peruviana L. antes y después del procesamiento. Los valores que no comparten la misma letra son diferentes, $p<0.05$.

\begin{tabular}{|l|c|c|}
\hline \multicolumn{1}{|c|}{ Condición } & Estado de madurez 3 & Estado de madurez 5 \\
\hline Fruta fresca & $237.3 \pm 5.8 \mathrm{a}$ & $260.7 \pm 5.8 \mathrm{~b}$ \\
\hline Fruta deshidratada por estufa & $110.7 \pm 5.8 \mathrm{D}$ & $130.7 \pm 5.8 \mathrm{C}$ \\
\hline Fruta deshidratada por secador de bandeja & $160.7 \pm 5.8 \mathrm{~B}$ & $184 \pm 10 \mathrm{~A}$ \\
\hline
\end{tabular}

La capacidad antioxidante presente en la fruta fresca es ligeramente superior al reportado por Puente et al. (2011) que fue de $210.82 \mu \mathrm{mol}$ TE/100 g muestra. Se puede observar que existe en promedio una degradación alta $(50 \%)$ de la capacidad ante el procesamiento de deshidratación por estufa, en comparación con el obtenido al deshidratar por secador de bandeja (31\%). Se conoce que el radical DPPH reacciona rápidamente con compuestos del tipo ácido ascórbico, ácido isoascórbico, isoeugenol, $\delta$-tocoferol (vitamina E), ácido Rosmarinico, ácido gálico y curcumina, pero también puede reaccionar con hidroquinonas, aminas aromáticas como la cisteína, por lo que la degradación de estos compuestos, sea por el tratamiento térmico realizado o las reacciones enzimáticas generadas, reducen la capacidad antioxidante como se observa en la tabla 2 (Molyneux, 2004; Mishra et al., 2012). Existe una alta correlación entre los polifenoles y la capacidad antioxidante de las frutas por lo antes descrito, sin embargo, se observa que debido a la capacidad del DPPH de reaccionar con otros compuestos, se explica que, aunque disminuye el contenido de polifenoles, aumenta la capacidad antioxidante en función de la maduración del fruto. 


\section{Contenido de Carotenos}

La tabla 3 muestra el contenido de carotenos presente en la fruta Physalis peruviana L. antes y después de su procesamiento con ambos secadores, donde se evidencia que existe un incremento del contenido de carotenos con el incremento de la madurez. Etzbach et al. (2018) encontraron este comportamiento al evaluar los carotenoides totales de Physalis peruviana L. durante distintos estados de madurez, y observaron que existe un incremento considerable desde $27.22 \mu \mathrm{g} / \mathrm{g}$ a $204.5 \mu \mathrm{g} / \mathrm{g}$ de fruta fresca entre el estado inmaduro al maduro, evidenciando que nuestros resultados se encuentran en el rango presentado por el mencionado autor. En ese mismo estudio se identificó que la fruta presentaba 20 distintos tipos de carotenoides mientras estaba en su etapa inmadura y 42 en su etapa madura, lo que explica igualmente el aumento progresivo de los carotenos durante la madurez de la fruta.

Tabla 3: Contenido de carotenos $(\mu \mathrm{g} / \mathrm{g})$ de Physalis peruviana $\mathrm{L}$. antes y después del procesamiento. Los valores que comparten la misma letra no son diferentes, $\mathrm{p}<0.05$.

\begin{tabular}{|l|c|c|}
\hline \multicolumn{1}{|c|}{ Condición } & Estado de madurez 3 & Estado de madurez 5 \\
\hline Fruta fresca & $136.96 \pm 5.46 \mathrm{~A}$ & $149.26 \pm 0.34 \mathrm{~A}$ \\
\hline Fruta deshidratada por estufa & $105.14 \pm 1.36 \mathrm{~b}$ & $116.23 \pm 4.77 \mathrm{~b}$ \\
\hline Fruta deshidratada por secador de bandeja & $123.94 \pm 11.59 \mathrm{a}$ & $132.62 \pm 8.87 \mathrm{a}$ \\
\hline
\end{tabular}

En la tabla 3 se evidencia también que existe una degradación de los carotenos ante el tratamiento térmico, con un porcentaje de degradación promedio de $22.68 \%$ en el secado con estufa y un $10.33 \%$ en el secado de bandejas. Esto permite evidenciar que los carotenos presentan una mayor resistencia al procesamiento térmico que los polifenoles o el ácido ascórbico. En la literatura se puede encontrar un decremento en el contenido de carotenos de Physalis peruviana L. entre 30 y $50 \%$, aunque en otros casos se ha evidenciado un incremento del contenido luego del tratamiento térmico (Olivares-Tenorio et al., 2016). Esto se debe a que durante los procesos térmicos se generan reacciones de isomerización y oxidación que degradan los carotenos, pero también los producen (Zepka y Mercadante, 2009).

\section{Contenido de Ácido Ascórbico}

La tabla 4 muestra los resultados del contenido de ácido ascórbico presente en la fruta de estudio en los dos estados de madurez tanto para la muestra fresca como para la muestra procesada en ambos equipos. Los resultados obtenidos se encuentran en el rango de $11-42 \mathrm{mg} / 100 \mathrm{~g}$ de peso fresco indicado por Fischer et al. (2014).

Tabla 4: Contenido de ácido ascórbico ( $\mathrm{mg} / 100 \mathrm{~g}$ peso fresco) de Physalis peruviana L. antes y después del procesamiento. Los valores que comparten la misma letra no son diferentes, $p<0.05$.

\begin{tabular}{|l|c|c|}
\hline \multicolumn{1}{|c|}{ Condición } & Estado de madurez 3 & Estado de madurez 5 \\
\hline Fruta fresca & $21.03 \pm 0.96 \mathrm{~A}$ & $19.10 \pm 0.79 \mathrm{~A}$ \\
\hline Fruta deshidratada por estufa & $9.16 \pm 0.36 \mathrm{~b}$ & $9.21 \pm 0.80 \mathrm{~b}$ \\
\hline Fruta deshidratada por secador de bandeja & $17.23 \pm 1.07 \mathrm{a}$ & $16.68 \pm 0.46 \mathrm{a}$ \\
\hline
\end{tabular}

Del análisis presente en la tabla 4 puede observarse que no existe diferencia estadística significativa al deshidratar la fruta en distintos estados de madurez, es decir, se degrada en igual manera el ácido ascórbico para la fruta en estado de madurez 3 que en el de 5. Este comportamiento se obtiene para los dos métodos de deshidratación, a pesar de esto si se evidencia una diferencia entre la deshidratación mediante estufa y la experimentada en secador de bandeja. En el primero el ácido ascórbico se degrada en un $54.11 \%$ en promedio, mientras que en la deshidratación por bandeja se degrada en un 15.37\%, el tiempo prolongado de tratamiento en el secador por estufa es el motivo de la alta degradación en comparación con el secador de bandejas. No obstante, en ambos casos existe una degradación dada la termosensibilidad del ácido a causa de la temperatura utilizada $\left(60^{\circ} \mathrm{C}\right)$ (Herbig y Renard, 2017). En estudios similares se obtuvo una mayor degradación (aproximadamente $85 \%$ ) para un intervalo de tiempo equivalente en el secador de estufa (Olivares-Tenorio et al., 2017). A pesar de este decaimiento, el contenido final de ácido ascórbico es relativamente alto y como indican Olivares-Tenorio et al. (2017), la estabilidad del ácido ascórbico puede deberse a la protección otorgada por compuestos antioxidantes como los carotenos o los fenoles, que pueden trabajar conjuntamente para protegerse de los procesos oxidativos durante el tratamiento térmico. 
Por otro lado, en la fruta fresca se evidencia una disminución del contenido de ácido ascórbico a medida que madura la fruta, este comportamiento se ha evidenciado también en otras frutas, tal es el caso del durazno donde el contenido de ácido ascórbico disminuyó de $0.32 \mathrm{~g} / 100 \mathrm{~g}$ de fruto inmaduro a $0.12 \mathrm{~g} / 100 \mathrm{~g}$ de fruto maduro (Cascales et al., 2005). Sin embargo, las letras iguales indican que no existe diferencia significativa en el contenido de ácido ascórbico de la materia prima para los estados de madurez evaluados en este estudio. Adicionalmente, se conoce que la capacidad antioxidante también se ve influenciada por el contenido de vitamina $C$ que pueda tener la fruta, lo que contribuye a la disminución de la capacidad antioxidante encontrada en este estudio.

\section{Análisis de color}

La tabla 5 muestra los resultados del análisis de color (en escala CIELab) que se realizó a la fruta antes y después del procesamiento térmico, a partir de los cuales se calculó el croma y el ángulo Hue. En esta tabla el tratamiento 1 corresponde a la fruta deshidratada en el secador de bandejas con madurez 3 , el tratamiento 2 corresponde a la fruta deshidratada en el secador de estufa con madurez 3 , el tratamiento 3 corresponde a la fruta deshidratada en el secador de bandejas con madurez 5 y por último el tratamiento 4 a la fruta deshidratada por secador de estufa con estado de madurez 5.

Tabla 5: Análisis de color en la escala CIELab de Physalis peruviana L. antes y después del procesamiento. Los valores que comparten la misma letra no son diferentes, $\mathrm{p}<0.05$.

\begin{tabular}{|l|c|c|c|c|c|}
\hline \multicolumn{1}{|c|}{ Estado } & $\mathrm{L}^{*}$ & $\mathrm{a}^{*}$ & $\mathrm{~b}^{*}$ & Croma & oHue \\
\hline Fruta fresca, madurez 3 & $66.63 \pm 1.04 \mathrm{~A}$ & $4.03 \pm 0.11 \mathrm{~B}$ & $58.95 \pm 1.15 \mathrm{~B}$ & $59.09 \pm 1.14 \mathrm{~B}$ & $86.09 \pm 0.16 \mathrm{~A}$ \\
\hline Fruta fresca, madurez 5 & $66.42 \pm 0.37 \mathrm{~A}$ & $7.16 \pm 0.55 \mathrm{~A}$ & $64.17 \pm 1.79 \mathrm{~A}$ & $64.57 \pm 1.74 \mathrm{~A}$ & $83.63 \pm 0.6 \mathrm{~B}$ \\
\hline Tratamiento 1 & $54.88 \pm 1.02 \mathrm{a}$ & $7.13 \pm 0.49 \mathrm{a}$ & $49.3 \pm 1.08 \mathrm{a}$ & $49.82 \pm 1.07 \mathrm{a}$ & $81.77 \pm 0.60 \mathrm{a}$ \\
\hline Tratamiento 2 & $52.67 \pm 1.55 \mathrm{bc}$ & $5.1 \pm 0.61 \mathrm{~b}$ & $39.84 \pm 1.44 \mathrm{c}$ & $40.17 \pm 1.49 \mathrm{c}$ & $82.72 \pm 0.66 \mathrm{a}$ \\
\hline Tratamiento 3 & $51.62 \pm 1.18 \mathrm{c}$ & $7.59 \pm 0.46 \mathrm{a}$ & $45.55 \pm 2.42 \mathrm{~b}$ & $46.07 \pm 2.44 \mathrm{~b}$ & $80.51 \pm 0.48 \mathrm{~b}$ \\
\hline Tratamiento 4 & $54.26 \pm 0.33 \mathrm{ab}$ & $5.94 \pm 0.24 \mathrm{~b}$ & $43.88 \pm 1.33 \mathrm{~b}$ & $44.28 \pm 1.3 \mathrm{~b}$ & $82.28 \pm 0.49 \mathrm{a}$ \\
\hline
\end{tabular}

Los valores de $L$, a y b obtenidos para la materia prima difieren con los encontrados en la literatura $(L=54.44$ $\pm 0.34, a=23.67 \pm 0.30$ y $b=59.85 \pm 0.29$ ), lo cual puede deberse a la madurez en la que fue evaluada o por la diferencia en los cultivares o ecotipos (Vega-Gálvez et al., 2014). Se observa adicionalmente que el valor de $L^{*}$ disminuye en un 17 a $22 \%$, luego de los procesos de deshidratación, esta disminución de la luminosidad está asociada con el oscurecimiento no enzimático y a la polimerización de los compuestos fenólicos (Reis et al., 2006). Por otro lado, si se observa el diagrama de cromaticidad en el espacio Lab, se puede identificar que los valores de la fruta deshidratada mediante estufa sin importar su madurez representan un color amarillo-anaranjado más oscuro en comparación con el de la fruta deshidratada en el secador de bandejas. En cualquier caso, todos los estados se encuentran en la región amarilla del diagrama de cromaticidad diferenciándose por el nivel de luminosidad y la intensidad del color.

\section{Correlaciones}

Finalmente, se realizó la correlación entre el ácido ascórbico, los carotenos y los polifenoles con la capacidad antioxidante de la materia prima, en función de conocer la relación de estos compuestos con la última variable. Esta correlación según Mukaka citado por Del'Arco y de Sylos (2018), es muy fuerte o muy alta si el valor está entre 0.9 a 1; alta o fuerte si el valor está entre 0.7 y 0.9 ; moderada de 0.5 a 0.7 ; baja de 0.5 a 0.3 y despreciable si la correlación es de 0 a 0.3 . En el caso de carotenos la correlación fue de 0.785 ; para el ácido ascórbico, la correlación fue de -0.777 , mientras que para los polifenoles la correlación fue de -0.841 . Lo que indica una correlación inversa entre los polifenoles y ácido ascórbico con la capacidad antioxidante. Esto puede explicarse por el incremento de la actividad de la enzima polifenoloxidasa durante la maduración de la fruta y el hecho de que el radical DPPH reacciona con compuestos adicionales a los polifenoles y ácido ascórbico.

En todos los casos la correlación es alta ya que estos compuestos poseen propiedades antioxidantes, sobre todo en los polifenoles y el ácido ascórbico los cuales se conoce reaccionan con el DPPH, ya que en su estructura química poseen anillos aromáticos con grupos hidroxilo que justifican el resultado obtenido. Adicionalmente, algunos carotenos reaccionan con el DPPH (aunque en menor medida) explicando también porque existe correlación entre estas dos variables, sin embargo, la capacidad antioxidante de una fruta es el resultado de la sinergia de diferentes componentes bioactivos presentes en la misma (Del'Arco y de Sylos, 2018). 


\section{CONCLUSIONES}

Del tratamiento térmico mediante dos distintos métodos de deshidratación (estufa y secador de bandeja) desarrollado al fruto Physalis peruviana L. con dos diferentes estados de madurez, se logró constatar las siguientes conclusiones: 1) tanto la cantidad de polifenoles totales como la capacidad antioxidante, ácido ascórbico, carotenos y color del fruto se degradan en mayor medida en el tratamiento mediante la estufa; 2) en cualquier caso, se evidenció que los compuestos con la menor degradación posterior al tratamiento térmico son los carotenos. 3) se evidenció que existe una disminución de los polifenoles totales y el ácido ascórbico, y un aumento de la capacidad antioxidante y los carotenos a medida que madura el fruto, por lo que se obtuvo una correlación negativa entre la capacidad antioxidante y los polifenoles y contenido de ácido ascórbico; y 4) se recomienda el uso del secador de bandejas para conservar en mejor medida las propiedades del fruto estudiado.

\section{REFERENCIAS}

Bolívar-Fernández, N., C. Saucedo-Veloz y E. Sauri-Duch, Respiración y Parámetros Relacionados Durante la Maduración del Chicozapote Cosechado en la Península de Yucatán, doi: 10.1590/S0100-29452011000500032, Rev. Bras. Frutic., 33(1), 261-266 (2011)

Bravo, K y E. Osorio, Characterization of Polyphenol Oxidase from Cape Gooseberry (Physalis peruviana L.) Fruit, doi: 10.1016/j.foodchem.2015.10.126, Food Chem., 197(A), 185-190 (2016)

Carriel, J.M., A.A. Obrebska, R. Bru-Martínez, M. Carranza, R. Pico-Saltos y E. Nieto, Distribución, Localización e Inhibidores de las Polifenol Oxidasas en Frutos y Vegetales Usados Como Alimentos, doi:10.18779/cyt.v7i1.95, Ciencia y Tecnología, 7(1), 23-31 (2014)

Cascales, A.I., E. Costell y F. Romojaro, Effects of the Degree of Maturity on the Chemical Composition, Physical Characteristics and Sensory Attributes of Peach (Prunus persica) cv. Caterin, doi: 10.1177/1082013205057943, Food Sci Tech Int, 11(5), 345-352 (2005)

Cerón, I., J.C. Higuita y C. Cardona, Capacidad Antioxidante y Contenido Fenólico Total de Tres Frutas Cultivadas en la Región Andina, ISSN: 1909-7891, Vector, 5, 17-26 (2010)

Copello, M.C., F. Villa y otros cinco autores, Bioactive Compounds, Antioxidant and Physic-Chemical Characteristics of the Dovyalis Fruit, doi: 10.4025/actasciagron.v40i2.35465, Acta Sci. Agron., 40, 1-8 (2018)

Corrales, A., A.I. Vergara, B. Rojano, E. Yahia y M.E. Maldonado, Características Nutricionales y Antioxidantes de la Uchuva Colombiana (Physalis peruviana L.) en tres Estadios de Maduración, ISSN: 0004-0622, Archivos Latinoamericanos de Nutrición, 65(4), (2015)

Cortés, G.M., G.A. Prieto y W.E. Rozo, Caracterización Bromatológica y Fisicoquímica de la Uchuva (Physalis peruviana L.) y su Posible Aplicación como Alimento Nutracéutico, ISSN: 0121-7488, Ciencia en Desarrollo, 6(1), 87-97 (2015)

de Torres, C., M.C. Díaz, I. Hermosín y M.S. Pérez, Effect of Freeze Drying and Oven Drying on Volatiles and Phenolics Composition of Grape Skin, doi: 10.1016/j.aca.2009.10.005, 660, Anal. Chim. Acta, (1-2), 177-182 (2010)

Del'Arco, A.P. y C.M. de Sylos, Effect of Industrial Processing for Obtaining Guava Paste on the Antioxidant Compounds of Guava (Psidium guajava I.) 'Paluma' cv., doi: 10.1590/0100-29452018011, Rev. Bras. Frutic. 40(2), 1-10 (2018)

Etzbach, L., A. Pfeiffer, F. Weber y A. Schieber, Characterization of Carotenoid Profiles in Goldenberry (Physalis peruviana L.) Fruits at Various Ripening Stages and in Different Plant Tissues by HPLC-DAD-APCI-MSn, doi: 10.1016/j.foodchem.2017.10.120, Food Chem., 245, 508-517 (2018)

Fischer, G., P.J. Almanza-Merchán y D, Miranda, Importancia y Cultivo de la Uchuva (Physalis peruviana L.), doi: 10.1590/0100-2945-441/13, Rev. Bras. Frutic., 36(1), 001-015 (2014)

Franco, A., C.S. Contreras, J. Carranza y J. Carranza, Total Phenolic Content and Antioxidant Capacity of Non-Native Wine Grapes Grown in Zacatecas, Mexico, ISSN: 1405-3195, Agrociencia, 51(6), 661-671 (2017)

Giraldo, G.I., C.D. Cruz y N.R. Sanabria, Propiedades Físicas del Jugo de Uchuva (Physalis peruviana) Clarificado en Función de la Concentración y la Temperatura, doi: 10.4067/S0718-07642017000100013, Inf. Tecnol., 28(1), 133-142 (2017)

Giraldo, R., T. Vargas y H. Gil, Mejoramiento del Proceso de Deshidratación de Uchuva, doi: 10.22490/25394088.567, Publicaciones e Investigación, 3(1), 37-49 (2009)

Gruz, J., F.A. Ayaz, H. Torun y M. Strnad, Phenolic Acid Content and Radical Scavenging Activity of Extracts from Medlar (Mespilus germanica L.) Fruit at Different Stages of Ripening, doi: 10.1016/j.foodchem.2010.06.030, Food Chem., 124, 271-277 (2011)

Gümüşay, Ö.A., A.A Borazan, N. Ercal y O. Demirkol, Drying Effects on the Antioxidants Properties of Tomatoes and Ginger, doi: 10.1016/j.foodchem.2014.09.162, Food Chem., 173, 156-162 (2015)

Herbig, A.L. y C.M. Renard, Factors that Impact the Stability of Vitamin C at Intermediate Temperatures in a Food Matrix, doi: 10.1016/j.foodchem.2016.10.012, Food Chem., 220, 444-451 (2017) 
Hoyos, J., M. Vásquez y J. Contreras, Electrochemical Methods as a Tool for Determining the Antioxidant Capacity of Food: A Review, doi:10.1016/j.foodchem.2016.11.017, Food Chem., 221, 1371-1381 (2017)

Mier H.J. y G. Cáez, Contenido de Polifenoles, Carotenos y Actividad Antioxidante en Frutos de Uchuva (Physalis peruviana) en Relación a su Estado de Maduración, ISSN: 1810-634X, Reciteia, 11(1b), 102-115 (2011)

Mishra, K., H. Ojha y N. K. Chaudhury, Estimation of Antiradical Properties of Antioxidants usin DPPH Assay: A Critical Review and Results, doi: 10.1016/j.foodchem.2011.07.127, Food Chem., 130, 1036-1043 (2012)

Molyneux, P., The Use of the Stable Free Radical Diphenylpicrylhydrazyl (DPPH) for Estimating Antioxidant Activity, ISSN: 2408-1779, Songklanakarin J. Sci. Technol., 26(2), 211-219 (2004)

Nunes, J.C., M.G. Lago y otros cinco autores, Effect of Drying Method on Volatile Compounds, Phenolic Profile and Antioxidant Capacity of Guava Powders, doi: 10.1016/j.foodchem.2015.11.050, Food Chem., 197, 881-890 (2016)

Olivares-Tenorio, M.L., M. Dekker, R. Verkerk y M. van Boekel, Health-Promoting Compounds in Cape Gooseberry (Physalis peruviana L.): Review from a Supply Chain Perspective, doi: 10.1016/j.tifs.2016.09.009, Trends in Food Science \& Technology, 57(A), 83-92 (2016)

Olivares-Tenorio, M.L., R. Verkerk, M.A. Boekel y M. Dekker, Thermal Stability of Phytochemicals, HMF and Antioxidant Activity in Cape Gooseberry (Physalis peruviana L.), doi: 10.1016/j.jff.2017.02.021, Journal of Functional Foods, 32, 4657 (2017)

Peinado J.L., R.V. Herrera, J.A. Grado y J.A. Gándara, Deshidratación de Alimentos Utilizando Energía Solar Térmica, ISSN: 2007-0411, CULCYT, 10(50), 99-107 (2013)

Puente, L.A., C.A. Pinto, E.S. Castro y M. Cortés, Physalis peruviana Linnaeus, the Multiple Properties of a Highly Functional Fruit: A Review, doi: 10.1016/j.foodres.2010.09.034, Food Research International, 44, 1733-1740 (2011)

Que, F., L. Mao, X. Fao y T. Wu, Comparison on Hot Air-Drying and Freeze-Drying on the Physicochemical Properties and Antioxidant Activities of Pumpkin (Cucurbita moschata Duch.) Flours, doi: 10.1111/j.1365-2621.2007.01590.x, Food Science \& Techonology, 43, 1195-1201 (2008)

Reis, R.C., A.M. Ramos, A.J. Regazzi, V.P. Minim y P.C. Stringueta, Almacenamiento de Mango Secado: Análisis Fisicoquímico, Microbiológico, Color y Sensorial, doi: 10.1080/11358120609487694, Ciencia y Tecnología Alimentaria, 5(3), 214-225 (2006)

Reyes-Beltrán, M., C.K. Guanilo-Reyes y otros cuatro autores, Efecto del consumo de Physalis peruviana L. (Aguaymanto) sobre el perfil lipídico de pacientes con Hipercolesterolemia, Acta Médica Peruana, ISSN: 1728-5917, 32(4), 195-201 (2015)

Sathyadevi, M. y S. Subramanian, Extraction, Isolation and Characterization of Bioactive Flavonoids from the Fruits of Physalis peruviana Linn Extract, Asian J. of Pharmaceutical and Clinical Research, ISSN: 2455-3891, 8(1), 152-157 (2015)

Scalzo, J., A. Politi, N. Pellegrini, B. Mezzetti y M. Battino, Plant Genotype Affects Total Antioxidant Capacity and Phenolic Contents in Fruit, doi: 10.1016/j.nut.2004.03.025, Nutrition, 21(2), 207-213 (2005)

Solorzano, S., T. Alia y otros siete autores, Quality Attributes and Functional Compunds of Mexican Plum (Spondias purpurea L.) Fruit Ecotypes, doi: 10.1051/fruits/2015027, Fruits, 70(5), 261-270 (2015)

Sun, Y., Y. Shen, D. Liu y X. Ye, Effects of Drying Methods on Phytochemical Compounds and Antioxidant Activity of Physiologically Dropped Un-Matured Citrus Fruits, doi: 10.1016/j.Iwt.2014.09.001, LWT - Food Science and Technology, 60(2), 1269-1275 (2015)

Teixeira, B.J., I.M. Aparcana y otros cinco autores, Evaluación del Contenido de Polifenoles Totales y la Capacidad Antioxidante de los Extractos Etanólicos de los Frutos de Aguaymanto (Physalis peruvian L.) de Diferentes Lugares del Perú, ISSN: 1810-634X, Rev. Soc. Quim., 82(3), 272-279 (2016)

Vega-Gálvez, A., J. López y otros cinco autores, High Hydrostatic Pressure Effect on Chemical Composition, Color, Phenolic Acids and Antioxidant Capacity of Cape Gooseberry Pulp (Physalis peruviana L.), doi: 10.1016/j.Iwt.2014.04.010, LWT-Food Science and Technology, 58(2), 519-526 (2014).

Wang, B., J. Wang y X. H. Zhao, In Vitro Activities of the Four Structurally Similar Flavonols Weakened by the Prior Thermal and Oxidative Treatments to a Human Colorectal Cancer Line, doi: 10.1111/jfbc.12310, Journal of Food Biochemistry, $41(2)$, e12310 (2017)

Yıldız, G., N. İzli, H. Ünal y V. Uylaşer, Physical and Chemical Characteristics of Goldenberry Fruit (Physalis peruviana L.), doi: 10.1007/s13197-014-1280-3, J. Food Sci. Technol., 52(4), 2320-2327 (2015)

Zepka, L.Q. y A.Z. Mercadante, Degradation Compounds of Carotenoids Formed during Heating of a Simulated Cashew Apple Juice, doi: 10.1016/j.foodchem.2009.03.071, Food Chem. 117, 28-34 (2009) 
